\title{
PRIME DECOMPOSITION OF KNOTS IN LORENZ-LIKE TEMPLATES
}

\author{
MIKE SULLIVAN
}

\begin{abstract}
In [7 R. F. Williams showed that all knots in the Lorenz template are prime. His proof included the cases where any number of positive twists were added to one of the template's branches. However [7] does give an example of a composite knot in a template with a single negative twist. Below we will show that in all the negative cases composite knots do exist, and give a mechanism for producing many examples. This problem was cited in a list of problems in dynamics in [1 problem 4.2].
\end{abstract}

\section{INTRODUCTION}

1.1. Flows, Knots and Templates. The periodic orbits of a flow in a 3-manifold form knots. These knots and how they are linked have been studied with the aid of templates or knots holders, i.e. 2-dimensional branched manifolds with semi-flows [4.

Definition 1. The $L(m, n)$ templates are shown in Figure [1 The semi-flow comes down from the branch line, $\beta$, forming two branches. The X-branch has $m$ half twists, while the Y-branch has $n$. The sign convention for the twists is that left-handed twists are positive and righthanded ones are negative. The two branches meet tangentially at the branch line.

$\tilde{L}(m, n)$ is the mirror image of $L(m, n)$.

The symbols defined above will also be used to represent the set of knots formed by the periodic orbits in the semi-flow of the template.

$L(0,0)$ is the Lorenz template [3]. The remainder will be called Lorenz-like templates. We also note that $L(m, n)=L(n, m)$.

\subsection{Prime and Composite Knots.}

Definition 2. A knot $k \subset S^{3}$ is composite if there exists a tame sphere $S^{2}$ such that $S^{2} \cap k$ is just two points, $p$ and $q$, and if $\gamma$ is any arc on

Date: October 30, 2018. 


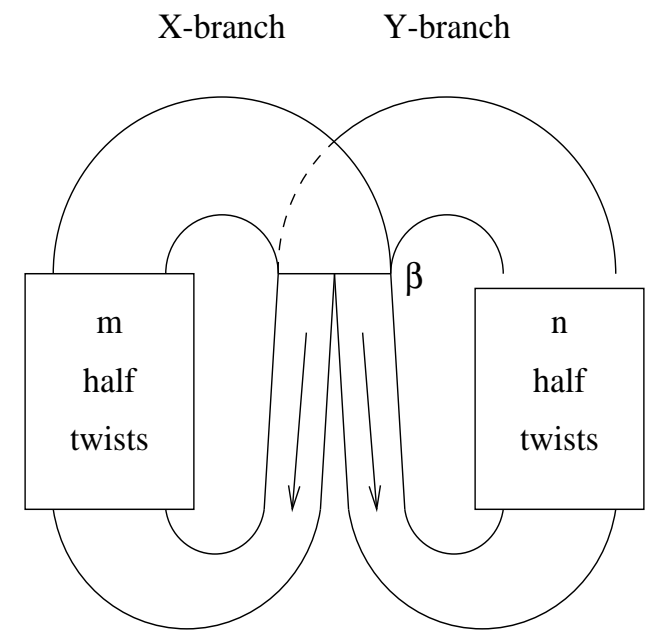

Figure 1. Lorenz-like template, $L(m, n)$, defined

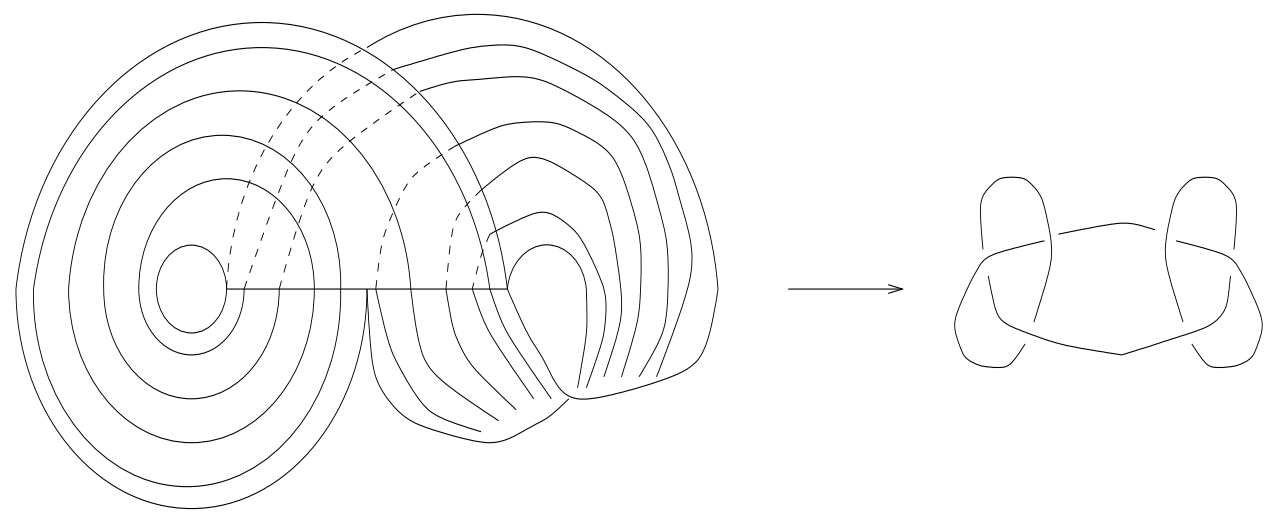

Figure 2. A square knot in $L(0,-1)$

$S^{2}$ joining $p$ to $q$, then the knots

$$
\begin{aligned}
& k_{1}=\gamma \cup\left(k \cap \text { outside of } S^{2}\right) \quad \text { and } \\
& k_{2}=\gamma \cup\left(k \cap \text { inside of } S^{2}\right),
\end{aligned}
$$

are nontrivial,(i.e. not the unknot). We call $k_{1}$ and $k_{2}$ factors of $k$ and write

$$
k=k_{1} \# k_{2} \text {. }
$$

If a knot isn't composite then it is prime.

Figure 3 gives an example.

Finally we state the all important theorem due to Schubert [6, 5]: 


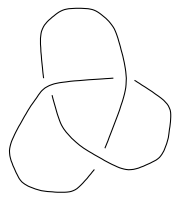

Left-hand Trefoil

Prime

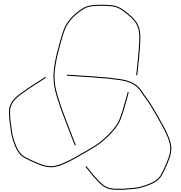

Right-hand Trefoil

Prime

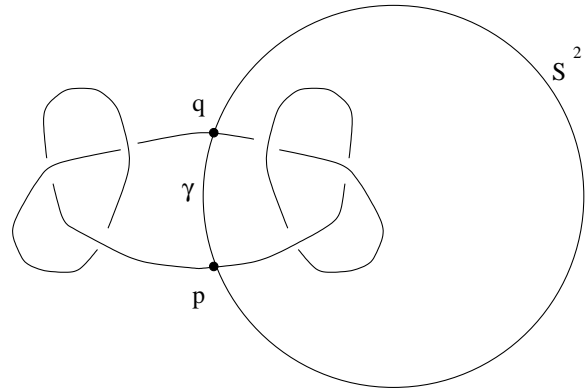

Square Knot

Composite

Figure 3. A connected sum

Knots can be factored uniquely into primes, up to order.

\section{KnOts IN $L(m, n)$}

Theorem 1. As sets of knots, $L(0, n-2) \supseteq L(0, n)$, for all integers $n$.

This may be restated as

$$
\begin{gathered}
\ldots \supseteq L(0,-2) \supset L(0,0) \supseteq L(0,2) \supseteq \ldots, \\
\ldots \supseteq L(0,-3) \supseteq L(0,-1) \supset L(0,1) \supseteq L(0,3) \supseteq \ldots,
\end{gathered}
$$

where the orientable and nonorientable cases are listed separately. We have also noted that two of the set inclusions are proper. Lorenz knots are positive braids [3], but $L(0,-2)$ has a negative braid. (To construct one, trace out the periodic orbit that wraps around the X-branch once and the Y-branch three times.) Knots in $L(0,1)$ are prime while $L(0,-1)$ has composite knots. It is not known which, if any, of the other set inclusions are proper.

Proof. Recall the equation "writhe = twist". We apply this in Figure 4 The illustration shows how to take a knot embedded in $L(0, n)$ over to a knot of the same knot type in $L(0, n-2)$.

Theorem 2. Any knot formed by the connected sum of a knot in $L(0,2)$ and a knot in $\tilde{L}(0,2)$ is in $L(0,-2)$.

Proof. The proof again is pictorial. We start with $L(-2,0)$, which is identical to $L(0,-2)$. By cutting along certain orbits and gradually deforming the template we will make the knots in question become clearly visible. 

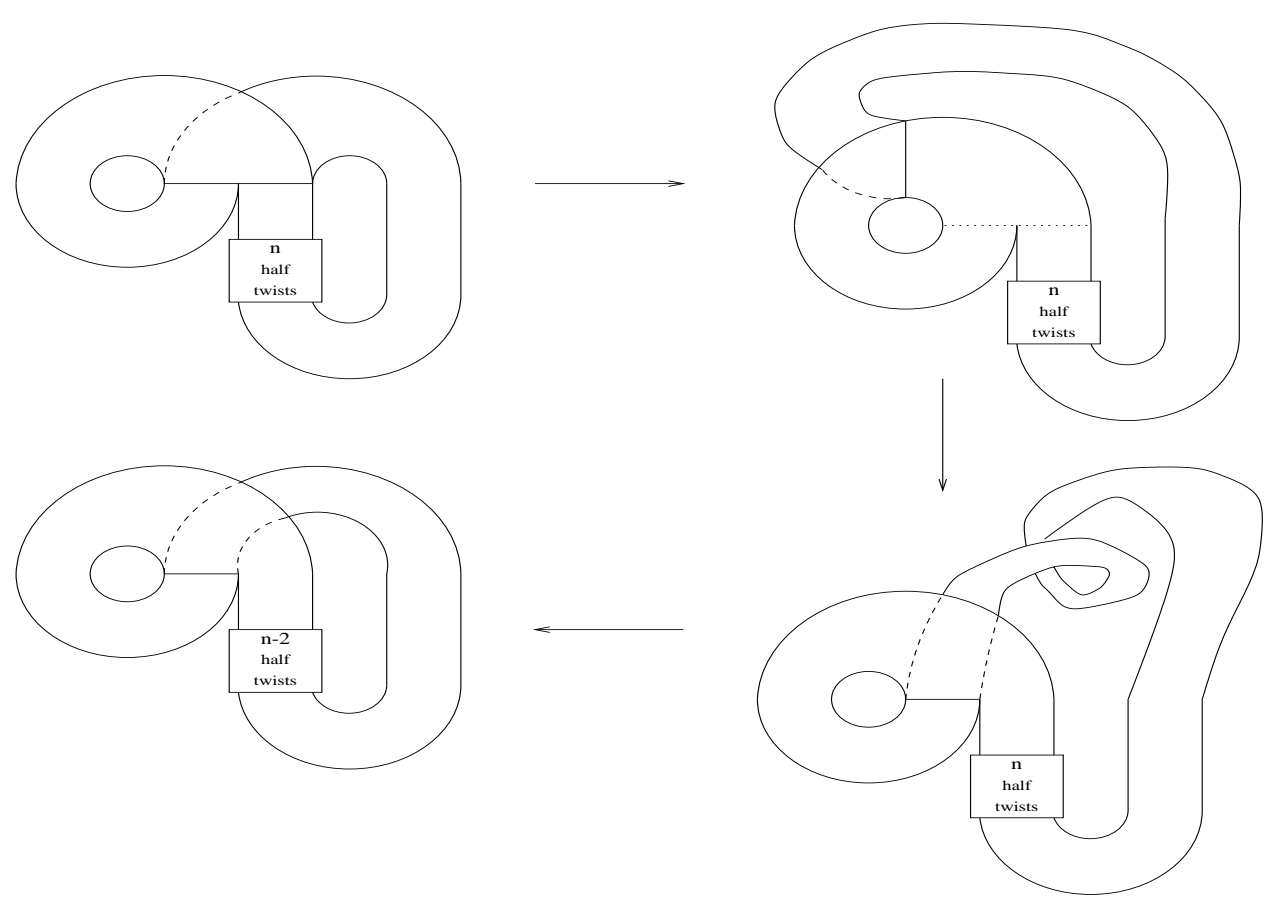

Figure 4. Proof of Theorem 1

Most of the steps in Figure 5 (a-w) should be self evident. Figures (a) to $(\mathrm{n})$ show that the subtemplate of $(\mathrm{n})$ lives in $L(-2,0)$. In going from figure $(\mathrm{j})$ to $(\mathrm{k})$ we have thrown away part of the template to help make the remaining steps clearer. The template of $(n)$ is shown in (o) with lines to cut along. This divides (o) into two parts, (p) and (t). To see that any pair of orbits on $(\mathrm{p})$ and $(\mathrm{t})$ respectively can be summed on (n) just study the example in Figure [6. Following from (p) to (s) we get $\tilde{L}(0,2)$. Likewise, (t) to $(\mathrm{w})$ gives $L(0,-2)$.

In Figure 6] we show a composite knot on (n) which is the square knot. In Figure 7 we display this knot on the original form of the template. The reader can check this by reversing all the steps while carrying the knot back. 
a)

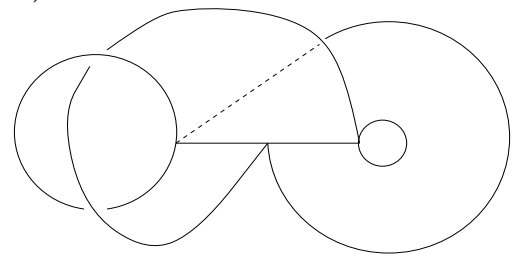

c)

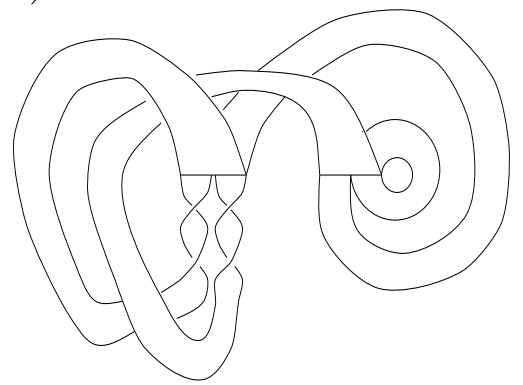

e)

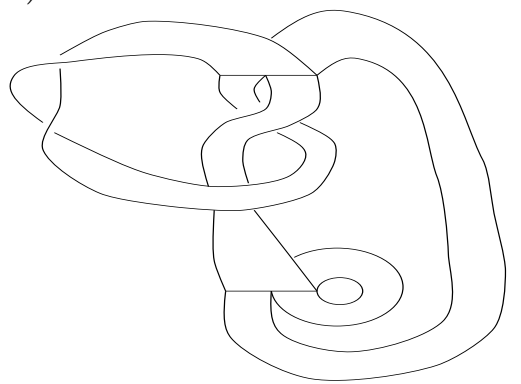

g)

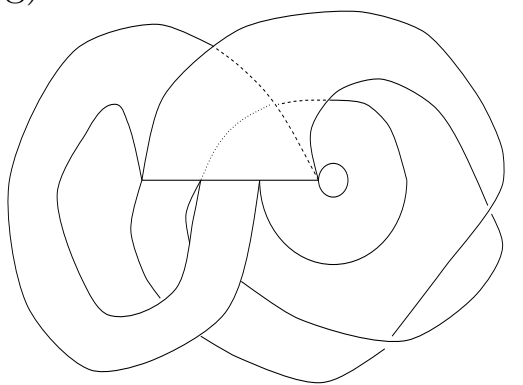

i)

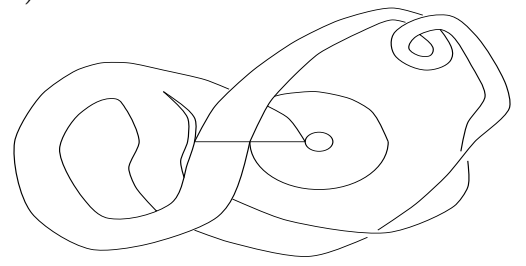

b)

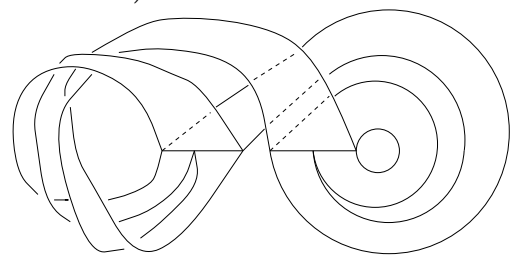

d)

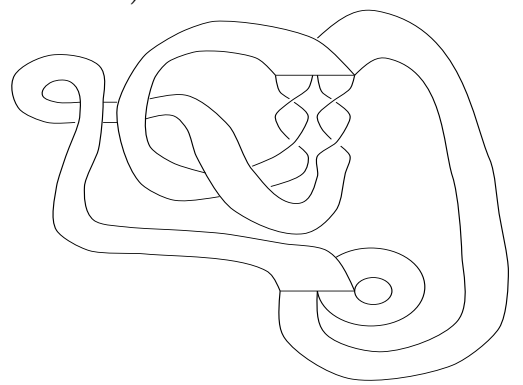

f)

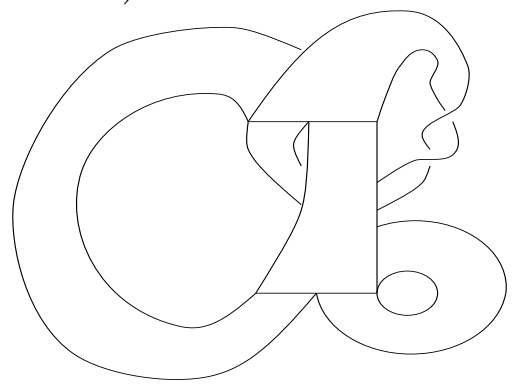

h)

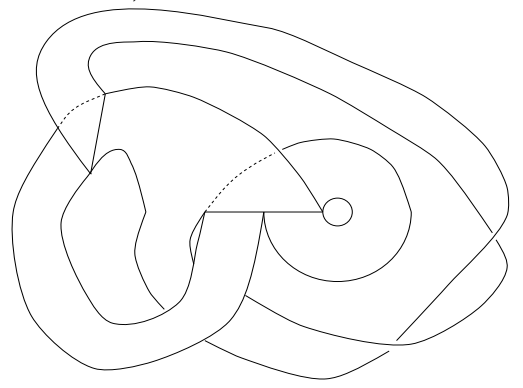

j)

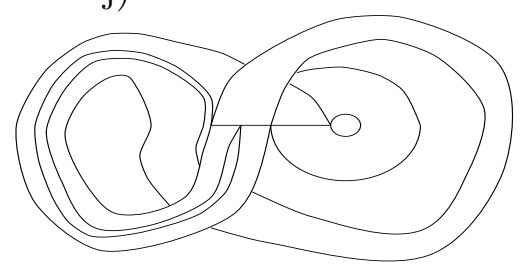


$\mathrm{k})$

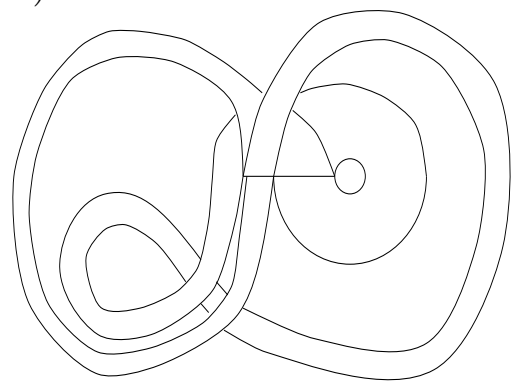

m)

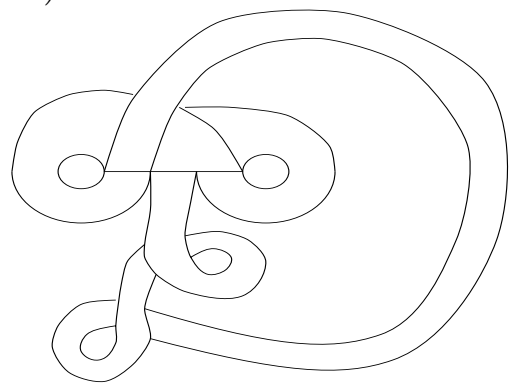

l)

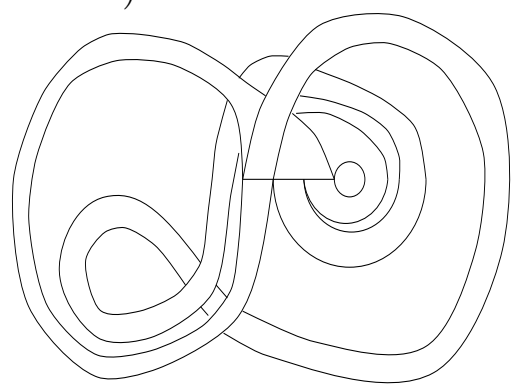

n)

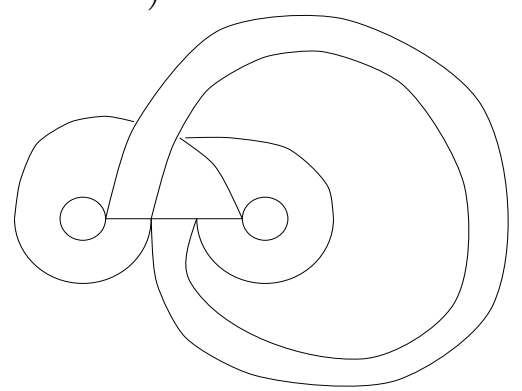

o)

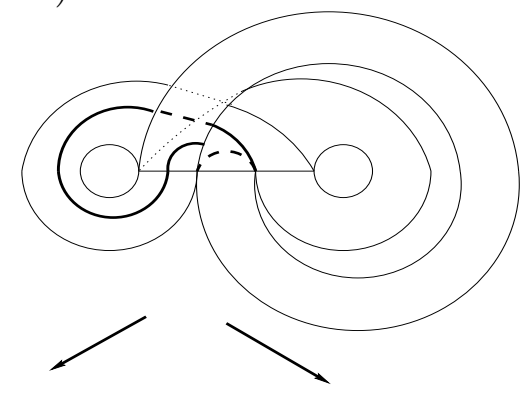

p)

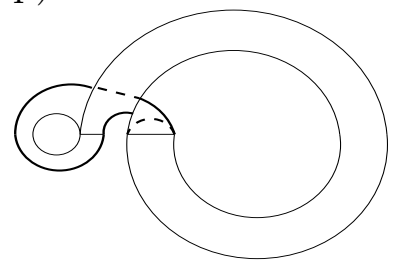

q)

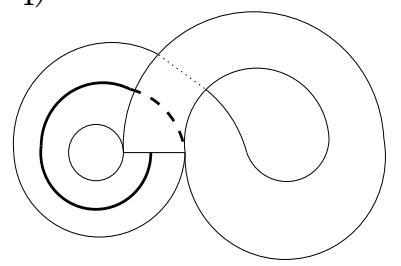

t)

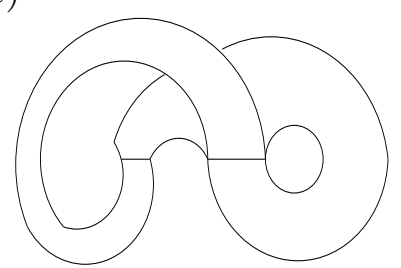

u)

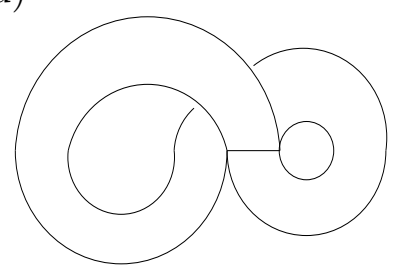


r)

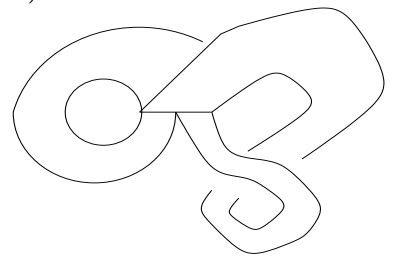

s)

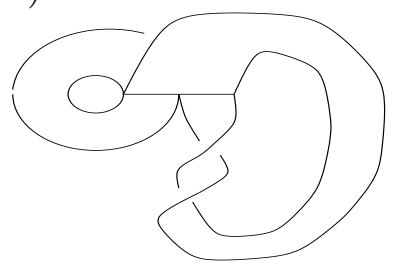

v)

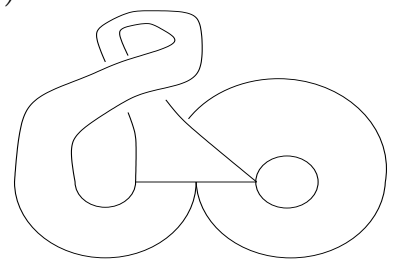

$\mathrm{w})$

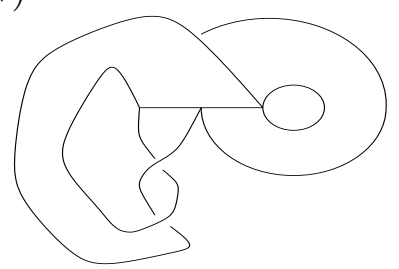

Figure 5. Figures (a)-(w) prove Theorem 2

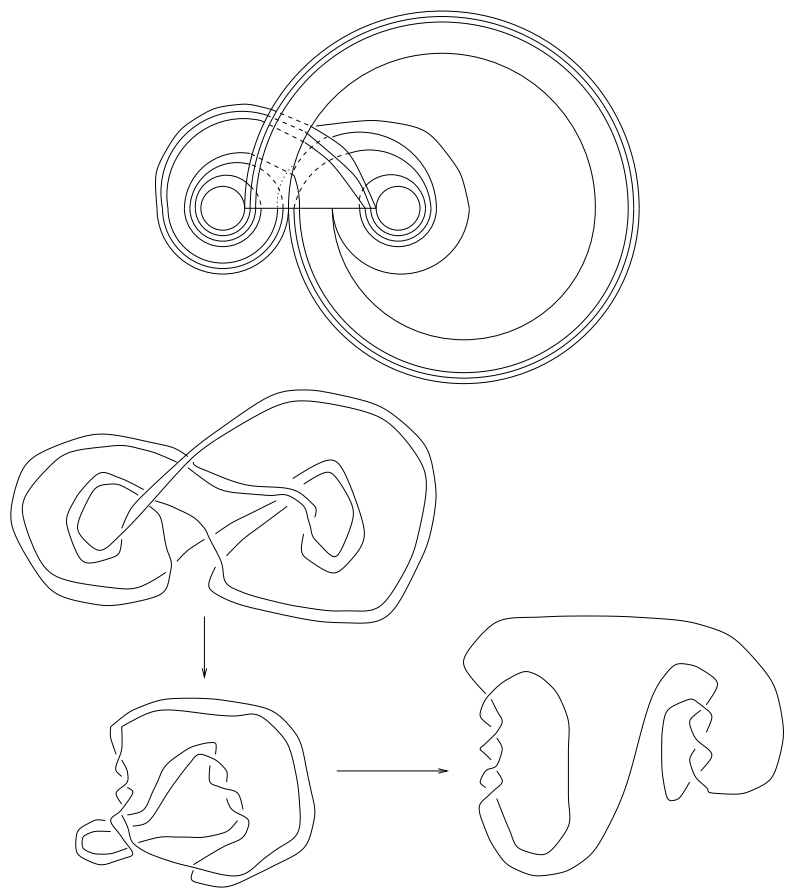

Figure 6. Composite knot

Thus TheOREMs 1 and 2 imply that for all negative integers $n$, $L(0, n)$ contains composite knots. In fact THEOREM 2 shows how to construct many examples of composite knots on $L(0,-2)$ and hence 


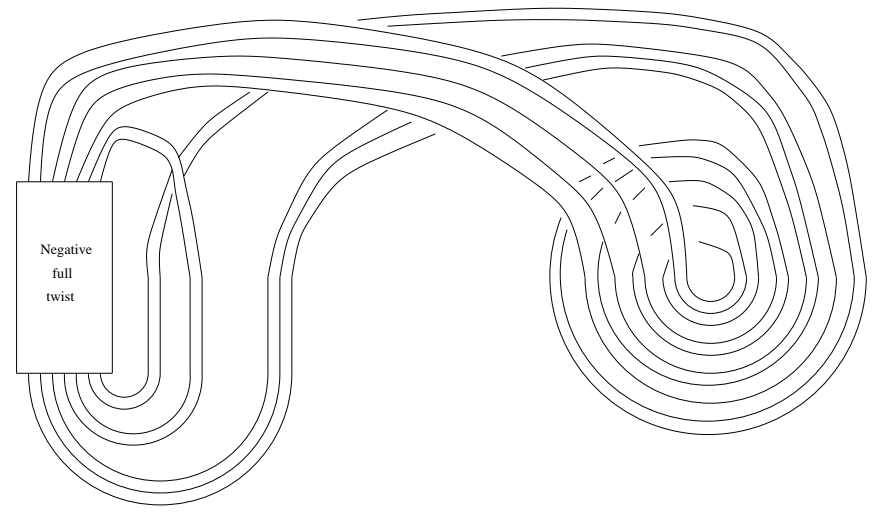

Figure 7. Composite knot on $L(-2,0)$

for any Lorenz-like template with an even number of negative twists on one its branches. The theorem below does this for the odd cases.

Theorem 3. As sets of knots $L(0,-4)$ and $\tilde{L}(1,-(2 n-1))$, for all $n>0$, are subsets of $L(0,-1)$.

Proof. Figure 8 shows that the knots in $L(0,-4)$ are in $L(0,-1)$. Figure 9 completes the proof.

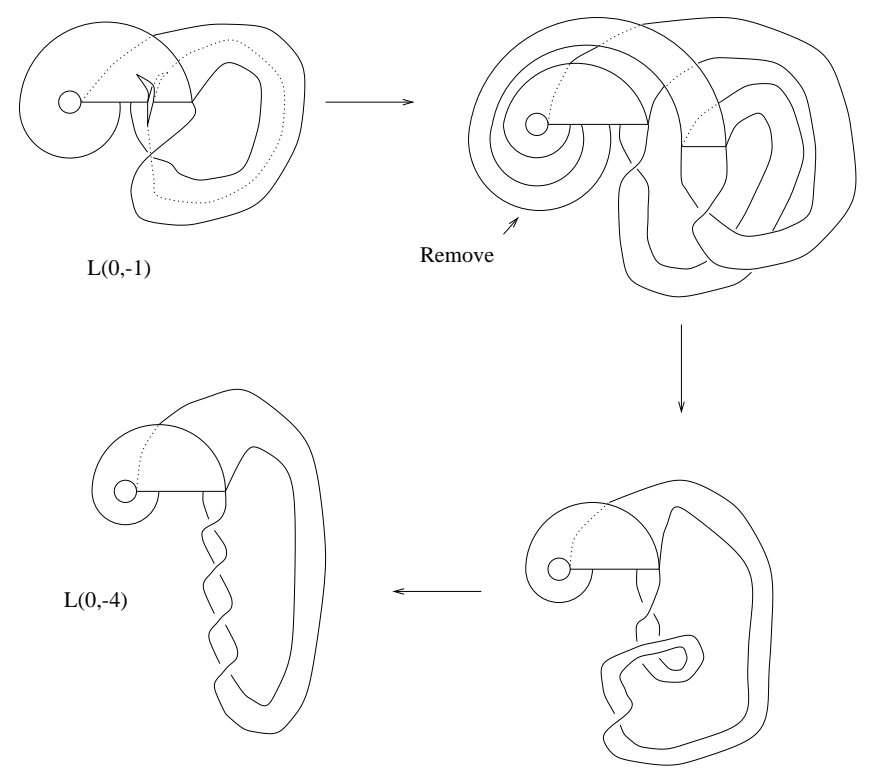

FiguRe 8. $L(0,-4) \subset L(0,-1)$ 
PRIME DECOMPOSITION OF KNOTS IN LORENZ-LIKE TEMPLATES 9

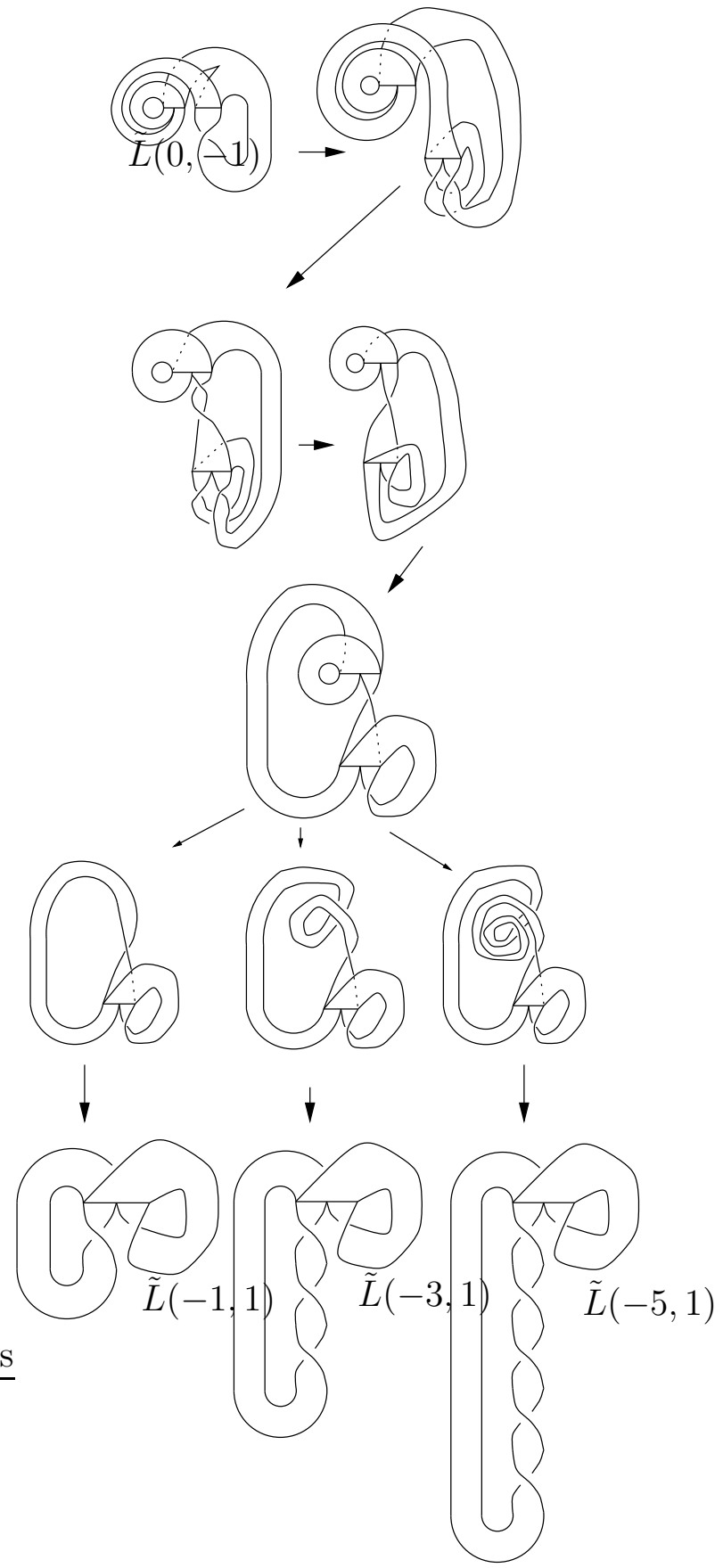

Figure 9. Rest of Theorem 3 
Acknowledgement: Supported in part by NSF grant no. DMS9001973.

\section{REFERENCES}

[1] M. Barge \& M. Brown Problems in Dynamics on Continua, Contemporary Mathematics: Continuum Theory and Dynamical Systems, 117, AMS, 1991.

[2] J. Birman. Braids, Links and Mapping Class Groups, Annals of Math. Studies \# 81, Princeton University Press, 1974.

[3] J. Birman \& R. Williams. Knotted Periodic Orbits in Dynamical Systems I: Lorenz Knots. Topology 22 (1983), 47-82.

[4] J. Birman \& R. Williams. Knotted Periodic Orbits in Dynamical Systems II: Knot Holders for Fibered Knots. Contemporary Math. 20 (1983).

[5] G. Burde \& H. Zieschang. Knots, Walter de Gruyter, 1985.

[6] H. Schubert. Knotten und Vollringe. Acta Math. 90 (1953), 131-286.

[7] R. Williams. Lorenz Knots are Prime. Ergod. Th. \& Dynam. Sys. 4 (1983), 147-163.

[8] R. Williams. Preprint: The braid index of generalized cables.

University of TeXas at Austin 